\title{
ÉVOLUTION RECENTE DES GLACIERS DU VIGNEMALE (2013-2017)
}

\author{
Recent evolution of the Vignemale \\ glaciers (2013-2017) \\ Simon Gascoin ${ }^{1, *}$, Pierre René ${ }^{2}$ \\ ${ }^{1}$ CESBIO, Université de Toulouse, CNES/CNRS/IRD/UPS, Toulouse, France. \\ ${ }^{2}$ Association Moraine, Luchon, France. \\ Identifiant ORCID des auteurs et e-mail: \\ Simon Gascoin: http://orcid.org/0000-0002-4996-6768. e-mail: simon.gascoin@cesbio.cnes.fr \\ Pierre René: https://orcid.org/0000-0002-5673-7971. e-mail: pierre.rene@wanadoo.fr \\ *Corresponding author
}

\begin{abstract}
Recibido: 05-02-2018. Aceptado: 03-04-2018. Fecha de publicación on-line: 29-05-2018
Citation / Cómo citar este artículo: Gascoin, S., René, P. (2018). Évolution recente des glaciers du Vignemale (2013-2017). Pirineos, 173, e037. https://doi.org/10.3989/pirineos.2018.173004

RESUME: Les glaciers d'Ossoue, du Petit Vignemale et des Oulettes sont les trois derniers glaciers du massif du Vignemale. Le plus grand d'entre eux, le glacier d'Ossoue, est l'un des mieux documentés dans les Pyrénées. Son évolution est un indicateur précieux des fluctuations du climat au sud-ouest de l'Europe. Une étude précédente de reconstruction du glacier d'Ossoue s'arrêtait en 2013 (Marti et al., 2015a). Nous présentons ici une mise à jour de son bilan de masse entre 2013 et 2017. Une carte de changement d'élévation a été produite à $4 \mathrm{~m}$ de résolution spatiale à partir de deux couples stéréoscopiques acquis par le système Pléiades à la fin des années glaciologiques 2013 et 2017. Les changements de hauteurs sont similaires à ceux mesurés par l'association Moraine au niveau de balises d'ablation sur la même période. Les données Pléiades permettent aussi d'estimer le bilan de masse des glaciers des Oulettes et du Petit Vignemale qui ne sont pas équipés de balises. Ainsi, entre 2013 et 2017, les bilans de masse des glaciers d'Ossoue, des Oulettes et du Petit Vignemale sont respectivement $-5,2+/-0,5 \mathrm{~m}$ we, $-4,0+/-0,9 \mathrm{~m}$ we et $-4,2+/-0,9 \mathrm{~m}$ we. Les données Pléiades montrent que le glacier d'Ossoue s'amincit plus rapidement au centre du Plateau des Neiges, ce qui peut s'expliquer par une accumulation de neige plus réduite dans cette zone par rapport aux bordures du glacier.
\end{abstract}

MOTS-CLES: Glacier; bilan de masse; télédétection; photogrammétrie.

ABSTRACT: The glaciers of Ossoue, Petit Vignemale and Oulettes are the last three glaciers of the Vignemale massif. The largest one, Ossoue glacier, is also one of the best documented in the Pyrenees. Its evolution is an important proxy of the climatic variations in southwest Europe. The latest reconstruction of the glacier evolution spanned the $20^{\text {th }}$ century up to 2013 (Marti et al., 2015a). Here we present an update of its mass balance between 2013 and 2017. An elevation change map was generated at $4 \mathrm{~m}$ resolution from two stereoscopic pairs, which were acquired by the 
Pléaides satellites system at the end of the summers 2013 and 2017. The elevation changes are similar to those that were routinely measured by the Moraine association at ablation stakes over the same period. In addition, the Pléiades data allow the estimation of the mass balance of Oulettes and Petit Vignemale glaciers, which are not equipped with stakes. We find that between 2013 and 2017, mass balance of the Ossoue, Oulettes and Petit Vignemale were respectively $-5,2+/-0,5 \mathrm{~m}$ we, $-4,0+/-0,9 \mathrm{~m}$ we and $-4,2+/-0,9 \mathrm{~m}$ we. Pléiades data further indicate that the thinning rate in Ossoue glacier is higher in the center of the "Plateau des Neiges" (the upper part of the glacier), which can be explained by lower snow accumulation in winter.

KEYWORDS: Glacier; mass balance; remote sensing; photogrammetry.

\section{Introduction}

Le massif du Vignemale dans les Pyrénées $\left(42^{\circ} 46^{\prime} 26^{\prime \prime} \mathrm{N}\right.$, $0^{\circ} 08^{\prime} 50^{\prime \prime} \mathrm{W}$ ) abrite trois glaciers, les glaciers d'Ossoue, du Petit Vignemale et des Oulettes. Les glaciers du Petit Vignemale et des Oulettes sont situés sur les pentes nord du massif et sont les reliques d'un même glacier qui s'est fragmenté à la fin du $19^{\text {e }}$ siècle (René, 2011; Marti et al., 2015a). Le glacier d'Ossoue prend sa source au-dessus de 3000 m d'altitude dans la dépression située entre le Vignemale et le Pic Central et s'écoule vers l'est. Il est actuellement le deuxième plus grand glacier pyrénéen, le plus grand du versant français (René, 2011; Rico et al., 2017). De plus, parmi les glaciers pyrénéens, le glacier d'Ossoue est sans doute celui dont on connaît le mieux l'évolution depuis la fin du Petit Âge Glaciaire, c'est-à-dire depuis 1850, grâce à de multiples documents d'archives (René 2013; Marti et al., 2015a). Par exemple, sa superficie est passée de 113 ha en 1850 à 45 ha en 2012 (Marti et al., $2015 b$ ). Cette réduction de surface cache néanmoins une évolution non linéaire au cours du 20 e siècle. Ainsi, le glacier d'Ossoue a connu une période de faible croissance entre les années 1950 et le début des années 1980 (René, 2011; Marti et al., 2015a). Depuis les années 1980, le glacier subit un retrait accéléré si bien qu'il est devenu un emblème des impacts du changement climatique dans les Pyrénées (René, 2013). En 2017 la fonte du glacier a conduit au détachement de la partie sud-est du glacier du corps principal, et la langue au nord-est est quasiment détachée (Figure 1).

L'association Moraine assure chaque année depuis 2002 la mesure du bilan de masse saisonnier du glacier d'Ossoue par la méthode glaciologique, c'est-à-dire à l'aide de 7 à 8 stations repérées (coordonnées géographiques) sur le glacier. Une autre estimation du bilan de masse sur la période 1983-2013 a été obtenue par méthode géodésique, c'est-à-dire par différence de modèles numériques d'élévation (MNE) (Marti et al., 2015a). Les deux MNE utilisés étaient issus de photographies aériennes multi-angulaires prises en 1983 et d'un couple d'images stéréoscopiques prises par le satellite Pléiades 1A. Comme la période utilisée pour le calcul du bilan de masse par ces deux méthodes était différente, il n'était pas possible de comparer les deux estimations.

Les images panchromatiques Pléiades ayant une résolution spatiale sub-métrique, les MNE dérivés de vues stéréoscopiques peuvent avoir une résolution spatiale de l'ordre du mètre au mieux (typiquement $2 \mathrm{~m}$ à $4 \mathrm{~m}$ ), avec une erreur aléatoire par pixel inférieure à $1 \mathrm{~m}$ (Berthier et al., 2014; Marti et al., 2016). Par différenciation de deux MNE Pléiades acquis entre deux dates suffisamment éloignées on peut donc produire une carte du bilan de masse d'un glacier avec une meilleure résolution spatiale que celle qui est permise par interpolation des mesures de balises. Cela permet aussi de caractériser l'évolution des glaciers les plus escarpés où il n'y a pas de balises (René, 2014).

A l'occasion du cinquantième anniversaire du Parc National des Pyrénées, un couple d'images stéréoscopiques du massif du Vignemale a été demandé par le Centre d'Etudes Spatiales de la Biosphère pour déterminer l'évolution des glaciers du Vignemale entre la précédente acquisition de 2013 et 2017 . Ce couple a été acquis le 8 octobre 2017 et nous a permis d'étudier les changements de surface et de hauteur des glaciers du Vignemale. Nous présentons dans cet article les résultats de cette étude ainsi qu'une comparaison avec les mesures de balises sur le glacier d'Ossoue. A partir de ces informations, nous dressons un bilan de l'évolution récente des glaciers du Vignemale.

\section{Données et méthodes}

\subsection{Bilan de masse glaciologique}

La méthode glaciologique couramment employée se scinde en deux campagnes de terrain distinctes. La première se déroule en fin de période d'accumulation (fin mai) et vise à mesurer la quantité de neige accumulée sur un glacier. Ce bilan hivernal est établi à partir de sondages et de carottages ponctuels, chacun représentatif d'une partie du glacier. La seconde investigation a lieu lorsque la saison d'ablation se termine (fin septembre) et permet de dresser le bilan estival de la fonte. Si la neige a entièrement fondu au niveau des stations de mesures alors des balises préalablement implantées dans la glace fournissent la hauteur de glace disparue (Tableau 1). Pour obtenir le bilan de masse moyen du glacier, les mesures de chaque station sont pondérées par la fraction de la surface du glacier occupée par la zone correspondante (Figure 2).

Les densités rencontrées dans un glacier sont variables du fait de sa composition (neige, névé, glace). Les carottages réalisés permettent d'en calculer les valeurs en prélevant des échantillons. Après conversion, l'unité employée pour le bilan est le mètre cube d'eau liquide que l'on divise souvent par la surface afin d'exprimer le bilan sous forme d'une lame d'eau gagnée ou perdue par le gla- 


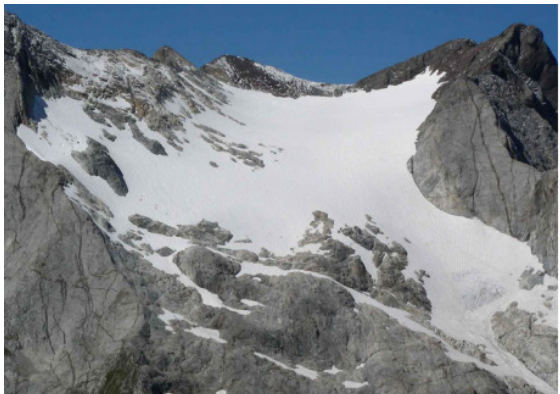

09-Sep-2013

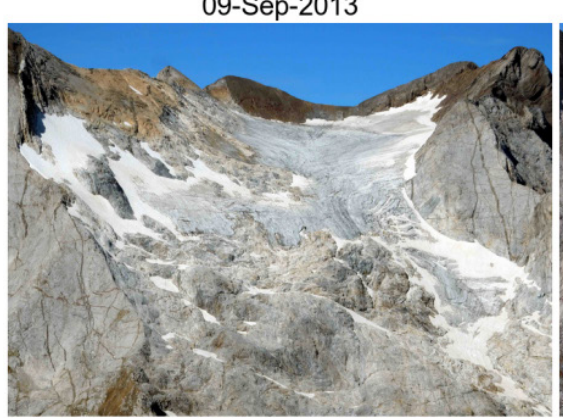

06-Sep-2016

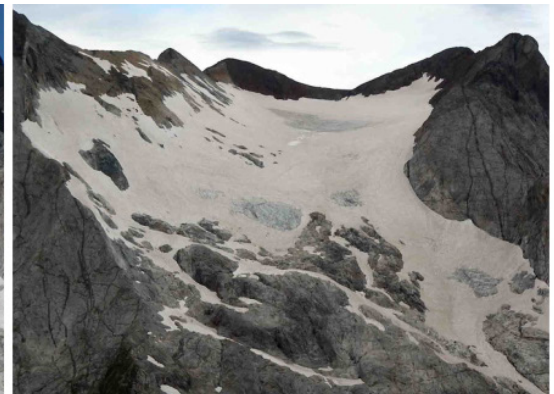

09-Sep-2014

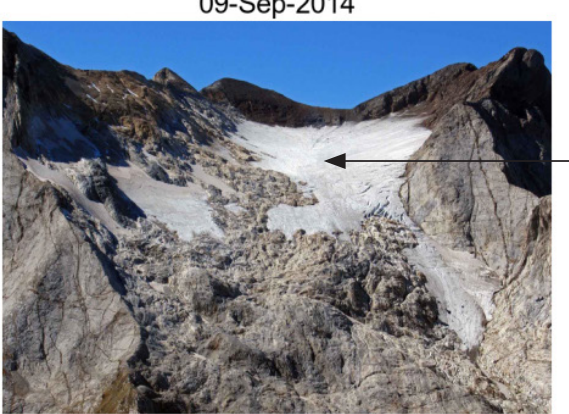

07-Oct-2017

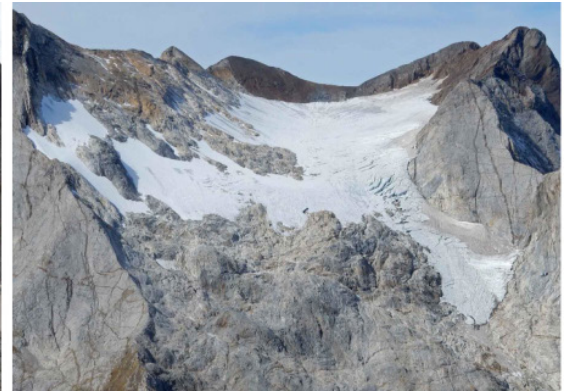

10-Oct-2015

Plateau des Neiges

Figure 1: Photographies du glacier d'Ossoue à la fin de l'été entre 2013 et 2017 (Association Moraine/Pierre René).

Figure 1: Late summer photographs of Ossoue Glacier between 2013 and 2017 (Association Moraine/Pierre René).

Tableau 1: Coordonnées géographiques des stations de mesures (balises) sur le glacier d'Ossoue et bilan des mesures effectuées entre le 06 octobre 2013 et le 08 octobre 2017 (changement de hauteur en m). La station 7 n'existe plus depuis 2016 en raison de la disparition de la zone associée. Le choix de l'unité (mètres en hauteur et pas mètres en équivalent en eau) est pour faciliter la comparaison avec le bilan géodésique.

Table 1: Geographic coordinates of the measurement stations in the Ossoue Glacier and summary of the measurements made between October 06, 2013 and October 08, 2017 (elevation changes in m). Station 7 no longer exists since 2016 due to the disappearance of the associated glacier area. The choice of the unit (meters in height and not meters in water equivalent) is to facilitate the comparison with the geodesic method.

\begin{tabular}{|c|c|c|c|c|c|}
\hline Station & Latitude & Longitude & Accumulation cumulée $(\mathbf{m})$ & Ablation cumulée $(\mathbf{m})$ & Bilan en fin d'été $(\mathbf{m})$ \\
\hline 1 & 42.77103 & -0.14814 & 23.7 & 28.5 & -4.8 \\
\hline 2 & 42.77222 & -0.14450 & 24.1 & 29.4 & -5.3 \\
\hline 3 & 42.77103 & -0.14450 & 18.0 & 26.9 & -8.9 \\
\hline 4 & 42.76992 & -0.14450 & 20.9 & 25.9 & -5.0 \\
\hline 5 & 42.77103 & -0.14089 & 19.8 & 28.8 & -9.0 \\
\hline 6 & 42.77111 & -0.13769 & 25.1 & 30.2 & -5.1 \\
\hline 8 & 42.77281 & -0.13564 & 25.3 & 38.1 & -12.8 \\
\hline
\end{tabular}

cier. Les valeurs mesurées sont ainsi indépendantes de la superficie est donc comparables entre les glaciers.

\subsection{Bilan de masse géodésique}

\subsubsection{Acquisitions}

Le premier couple d'images stéréoscopiques a été acquis le 23 septembre 2013 par Pléiades 1A dans le cadre de la recette thématique utilisateur coordonnée par le Centre National d'Études Spatiales (Tableau 2). Le second couple a été acquis le 8 octobre 2017 dans le cadre du programme ISIS (Figure 3). La résolution native des images panchromatiques est proche de $70 \mathrm{~cm}$ mais les images sont fournies après rééchantillonnage à une résolution de $50 \mathrm{~cm}$. Des images multispectrales (résolution $2.5 \mathrm{~m}$ ) ont été acquises également mais n'ont pas été exploitées ici.

\subsubsection{Traitement des images}

Dans l'étude de Marti et al. (2014), les images Pléiades avaient été traitées avec le logiciel Geoview de 


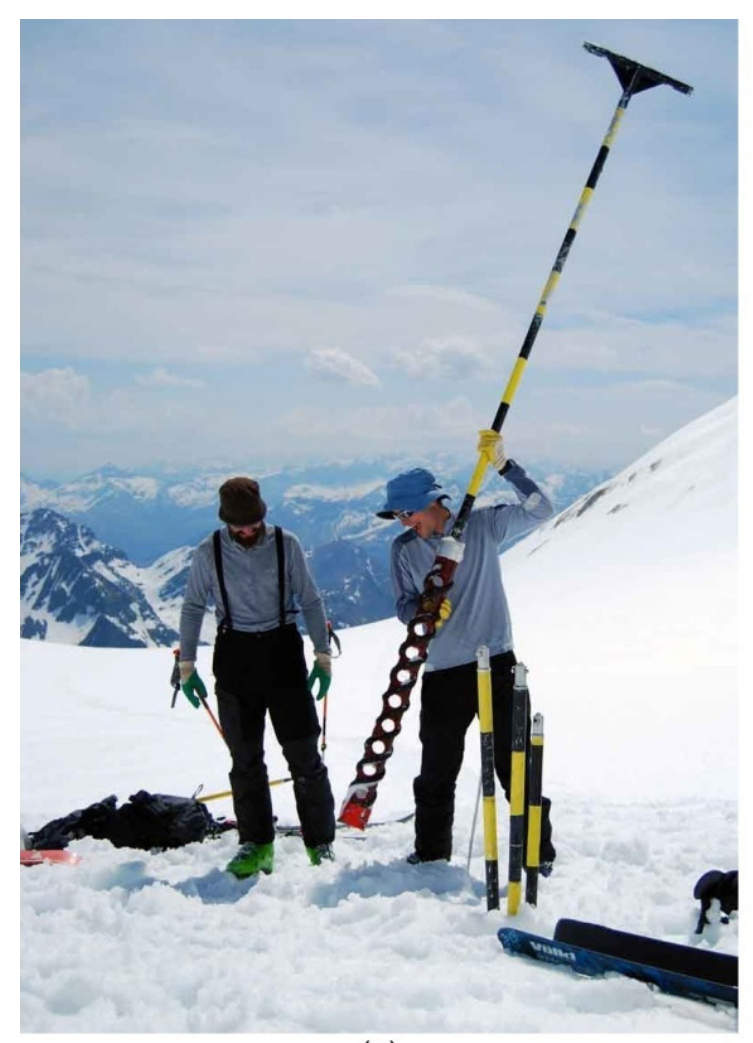

(a)

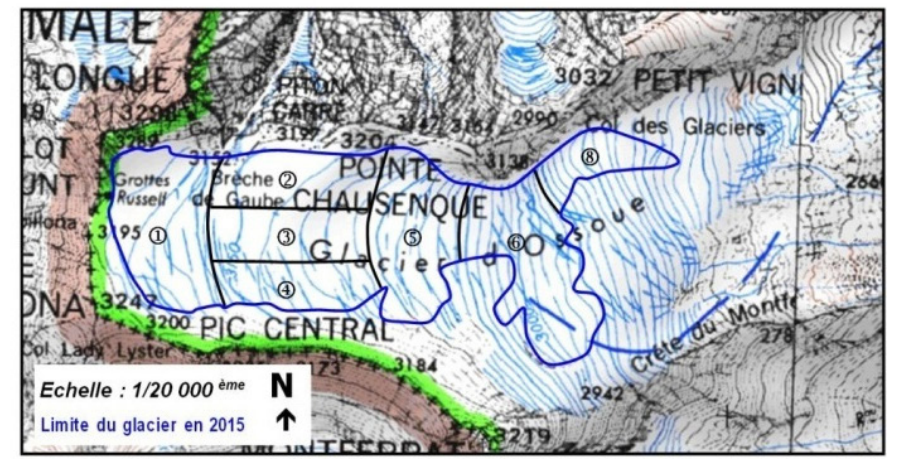

(b)

Figure 2: (a) carottage sur le glacier d'Ossoue; (b) position des stations de mesures et zonage associé pour le calcul du bilan de masse.

Figure 2: (a) Drilling a snow core on Ossoue Glacier; (b) location of the measurement stations (ablation stakes) and associated zoning for the glacier-wide mass balance calculation.

Tableau 2: Caractéristiques des couples stéréoscopiques d'images Pléiades utilisé pour cette étude. Le rapport B/H est calculé à partir de la différence entre les angles d'incidence along-track pris au centre de l'image.

Table 2: Characteristics of the stereoscopic pairs of Pléiades images used for this study. The B/H ratio is calculated from the difference between the incidence angles along-track taken in the center of the images.

\begin{tabular}{|l|c|c|}
\hline Date d'acquisition & $23 / 09 / 2013$ & $08 / 10 / 2017$ \\
\hline Plateforme & $1 \mathrm{~A}$ & $1 \mathrm{~B}$ \\
\hline B/H & 0.42 & 0.39 \\
\hline Emprise lon/lat $\left(^{\circ}\right)$ & $-0.25,42.67 ;-0.02,42.86$ & $-0.22,42.72 ;-0.04,42.82$ \\
\hline
\end{tabular}

l'Institut Géographique National (IGN). Ce logiciel a donné des résultats satisfaisants pour la surface du glacier d'Ossoue, mais une contrainte importante était que le logiciel ne permettait pas de traiter des images codées avec une profondeur radiométrique de 8 bits (256 valeurs). Comme les images Pléiades sont codées sur 12 bits (2048 valeurs), il avait donc fallu réduire la dynamique de l'image. Cela avait été fait au détriment des zones à l'ombre. Pour palier à cette limitation, les images de 2013 ont été retraitées pour cette étude avec la suite photogrammétrique Ames Stereo Pipeline (ASP; Shean et al., 2016). Cet outil utilise la dynamique complète des images Pléiades dans le processus de restitution photogrammétrique de l'élévation. De plus, l'utilisation d'ASP permet d'améliorer la reproductibilité des résultats puisque le logiciel est open source.

Le logiciel ASP a permis de produire des MNE à partir d'images Pléiades de glaciers ou de surfaces enneigées en zone de montagne ayant une erreur aléatoire verticale inférieure à $1 \mathrm{~m}$ (Berthier et al., 2014, Marti et al., 2016). De plus, l'erreur sur la différence de deux MNE Pléiades peut être réduite à $50 \mathrm{~cm}$ après co-registration des deux MNE (Marti et al., 2016; Belart et al., 2017). La version d'ASP utilisée pour cette étude est la 5.2.1. L'outil stereo d'ASP a été exécuté avec la même configuration que celle présentée par Marti et al. (2016). Le nuage de points a été converti en MNE avec l'outil point2dem sur une grille de $4 \mathrm{~m}$ de résolution spatiale dans la projection Lambert-93. 


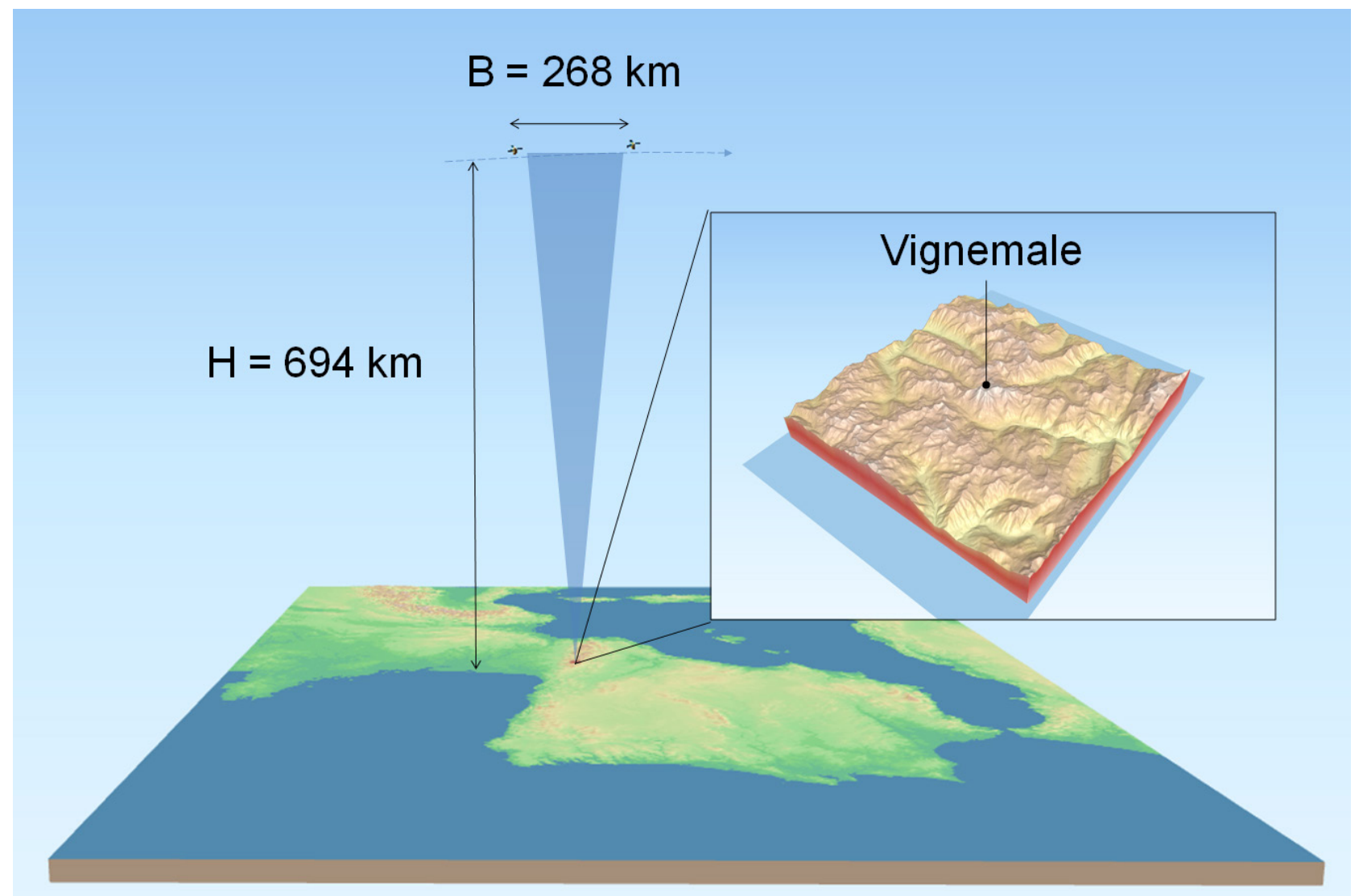

Figure 3: Géométrie d'acquisition de la paire d'images stéréoscopiques Pléiades 1B du 8 octobre 2017. Les distances B (base) et $\mathrm{H}$ (hauteur) sont à l'échelle. L'encadré illustre le relief de la zone imagée.

Figure 3: Acquisition geometry of the pair of Pléiades $1 B$ stereoscopic images of October 8, 2017. The distances B (base) and $H$ (height) are drawn to the same scale. The inset illustrates the relief of the imaged area.

Une ortho-image à $50 \mathrm{~cm}$ de résolution a été générée avec ce même outil en utilisant le MNE obtenu et une des images Pléiades (ici on a pris la vue avant). Les images de 2017 ont été traitées de façon strictement identique.

Une fois les deux MNE générés, nous les avons co-registrés sur sur le MNE de l'Institut Géographique National Espagnol (Modelo Digital del Terreno 5 metros de España, MDT05). Ce MNE a une résolution horizontale de $5 \mathrm{~m}$ et a été produit à partir de mesures Lidar aéroportées en 2010. La partie du MDT05 qui couvre l'emprise des images Pléiades a été reprojetée dans le système Lambert-93 par rééchantillonnage bicubique à $5 \mathrm{~m}$ de résolution. L'emprise des MNE Pléiades n'est que partiellement couverte par le MDT05 car il ne s'étend pas au delà de quelques $\mathrm{km}$ de la frontière sur le territoire français. Les MNE Pléiades ont néanmoins été co-registrés sur cette base car des tests préalables nous ont montré que ce MNE est de meilleure qualité sur notre zone d'étude que le MNE distribué par l'IGN français (référentiel à grande échelle, RGE).

La co-registration tridimensionnelle des MNE Pléiades sur le MDT05 a été effectuée pour chaque MNE par une translation en deux étapes séquentielles (Berthier et al.,
2007). Cette méthode a donné des résultats performants avec des MNE Pléiades en zone de montagne (e.g. Marti et al., 2016). D'abord une translation horizontale est effectuée, dont les composante sont calculées par minimisation de l'écart-type des différences d'élévations sur les pixels du MDT05 ayant une pente est comprise entre $4^{\circ}$ et $60^{\circ}$. Ensuite une translation verticale est effectuée, dont la composante est la médiane de la différence des élévations sur les zones du MDT05 ayant une pente inférieure à $45^{\circ}$. Les zones englacées sont également exclues dans les deux étapes par application du masque de glacier disponible dans le Randolph Glacier Inventory 5.0 (Arendt et al., 2015). Les polygones des glaciers du massif du Vignemale ont été produits par Marti et al. (2015b) à partir d'ortho-images aériennes acquises en juillet 2012 par l'IGN français. Pour évaluer le résultat du recalage nous avons calculé le NMAD (déviation absolue médiane normalisée, Höhle \& Höhle, 2009) des différences d'élévation hors des zones englacées. Cet indice est une mesure robuste de la dispersion des résidus. Le NMAD est inférieur à $1 \mathrm{~m}$ ce qui indique un bon recalage vis à vis du MDT05 (Tableau 3). Les composantes horizontales de la translation (Tableau 3) sont conformes aux spécifications du système Pléiades, 
Tableau 3: Paramètres et résultats du recalage des MNE Pléiades sur le MDT05. Le NMAD, la déviation absolue médiane normalisée, est un indicateur de la dispersion des résidus de la différence entre chaque MNE Pléiades et le MDT05 (cf Méthodes). Table 3: Parameters and results of the registration of Pléiades digital elevation models (DEM) on the MDT05 DEM. The normalized median absolute deviation (NMAD), is an indicator of the dispersion of the difference between each Pléiades DEM and the MDT05 (cf Methods).

\begin{tabular}{|c|c|c|}
\hline Année du MNE & Décalage en X; Y (m) & NMAD après recalage (m) \\
\hline 2013 & 13,$69 ; 6.03$ & 0,84 \\
\hline 2017 & $1.72 ; 0.11$ & 0,75 \\
\hline
\end{tabular}

puisque l'erreur circulaire de géolocalisation horizontale CE90 inférieure à $6.5 \mathrm{~m}$ (Gleyzes et al., 2012).

Enfin, le NMAD hors glacier calculé entre les deux MNE Pléiades est $0,56 \mathrm{~m}$ et $0,47 \mathrm{~m}$ si on exclut les différences aberrantes supérieures à $100 \mathrm{~m}$. Comme ces erreurs aberrantes ne sont pas présentes sur le glacier d'Ossoue, on peut estimer que l'erreur aléatoire sur la différence d'élévation sur les glaciers est également de l'ordre de $0,50 \mathrm{~m}$. La médiane des différences est $-0,04 \mathrm{~m}$, ce qui indique un biais résiduel négligeable. Cette incertitude de $0,50 \mathrm{~m}$ est cohérente avec les études précédemment publiées mais elle pourrait être sous-estimée pour les glaciers des Oulettes et du Petit Vignemale qui sont à l'ombre des parois et donc moins favorables à une bonne restitution du relief par la méthode stéréoscopique que le reste des images. Pour ces deux glaciers on utilise donc comme incertitude des résultats de changement d'élévation le double de la valeur précédemment calculée (soit $1 \mathrm{~m}$ ).

Nous avons ensuite découpé la carte des différences de MNE avec les polygones des glaciers de 2012. Les images Pléiades de 2013 ne permettent pas de déterminer l'étendue du glacier en raison d'une couche de neige qui masque les bords du glacier. L'évolution récente du glacier suggère que la surface du glacier en 2013 était vraisemblablement inférieure à celle de 2012. Par conséquent, on suppose que le polygone de 2012 utilisé pour découper la carte de changement d'élévation inclut la surface réelle du glacier en 2013. En revanche, les images Pléiades de 2017 permettent de déterminer la surface du glacier d'Ossoue. Pour cela, l'ortho-image de 2017 a été translatée avec les composantes horizontales de la translation appliquée au MNE et le contour du glacier a été digitalisé manuellement. Un tampon avec une distance fixe de $+/-5 \mathrm{~m}$ a été utilisé pour estimer l'incertitude sur la superficie du polygone.

Marti et al. (2014) estiment par interpolation des mesures de balises que $42 \mathrm{~cm}$ de hauteur neige ont fondu entre la date d'acquisition des images Pléiades le 23 septembre et le 6 octobre 2013 considéré comme la fin de l'année hydrologique en 2013.

\section{Résultats}

\subsection{Evolution de la superficie des glaciers entre 2011 et 2017}

La Figure 4 montre l'évolution de la superficie du glacier d'Ossoue entre 2011 et 2017. Sa superficie déterminée à partir de l'ortho-image Pléiades du 8 octobre 2017 est de 32,9 ha +/- 2,0 ha. La partie avale de la langue orientale s'est détachée du glacier pour former un nouveau glacieret d'une superficie de 2,7 +/- 0,5 ha (Figure 4).

Pour le glacier des Oulettes il est difficile de déterminer la superficie du glacier à partir de l'ortho-image Pléiades en raison d'un éclairement insuffisant. De plus, la partie basse du glacier est couverte de débris et donc difficile à cartographier à partir d'images satellite. Les relevés de terrain effectués par l'association Moraine indiquent pour 2009: 13 ha; 2012: 13 ha; 2015: 11 ha; 2016: 11 ha. En revanche, la surface du glacier du Petit Vignemale est similaire à celle de 2011.

\subsection{Evolution de la topographie des glaciers entre 2013 et 2017}

\subsubsection{Validation de la variation de hauteur du glacier d'Ossoue avec les mesures de balises}

Comme le montre la Figure 5, les variations de hauteur mesurées aux balises entre le 6 octobre 2013 et le 8 octobre 2017 sont en bon accord avec celles déterminées par la méthode géodésique (coefficient de corrélation linéaire $\mathrm{R}=0.97$, $\mathrm{p}$-value $=3 \mathrm{e}-4)$.

\subsubsection{Bilan de masse géodésique}

Entre le 23/09/2013 et le 08/10/2017 la surface du glacier d'Ossoue s'est abaissée en moyenne de -6,20 +/$0,5 \mathrm{~m}$ (Figure 6). En retirant la couche de neige ayant fondu entre l'acquisition de 2013 et la fin de l'année hydrologique (estimée au 6 octobre 2013), ce changement de hauteur correspond à une perte de glace dont la densité peut être fixée à $900 \mathrm{~kg} \cdot \mathrm{m}^{-3}$. Cela permet de calculer un bilan de masse de $-5,20+/-0,5 \mathrm{~m}$ we entre le 6 octobre 2013 et le 8 octobre 2017, soit une perte de masse annuelle de $-1,30 \mathrm{~m}$ we. $\mathrm{a}^{-1}$. A l'échelle du glacier et sur la même période, le bilan de masse du glacier d'Ossoue par la méthode glaciologique est $-6.35 \mathrm{~m}$ we. Le centre du Plateau des Neiges s'est davantage aminci que les bords du glacier (Figure 6). Dans ce secteur, le glacier a perdu plus de $2 \mathrm{~m}$ de hauteur de glace par an au cours des quatre dernières années.

Pour le glacier des Oulettes, la variation moyenne de hauteur entre les deux acquisitions Pléiades est de -4,46 

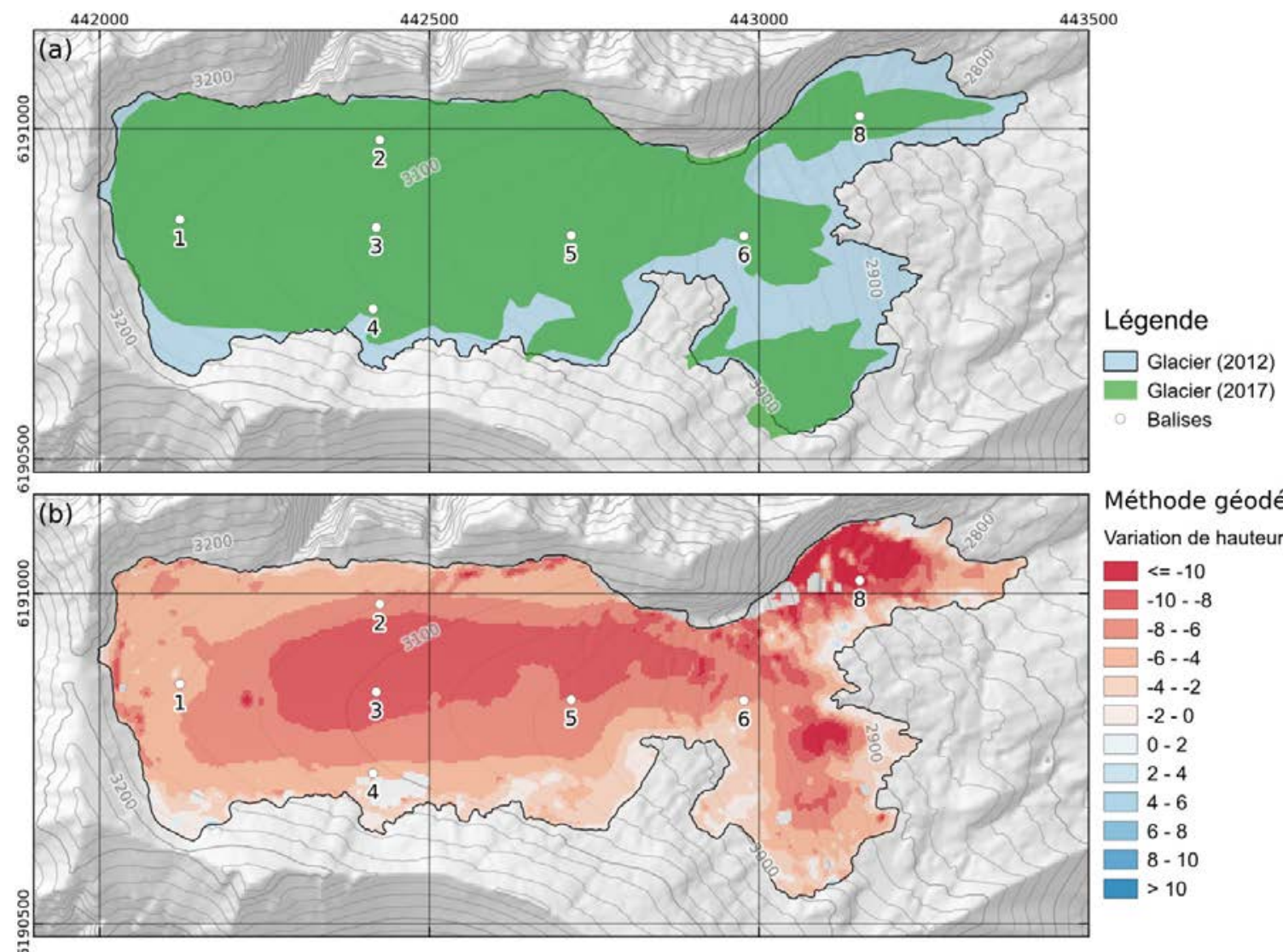

\section{Méthode géodésique}

Variation de hauteur $(\mathrm{m})$ 2013-2017

C $<=-10$

$-10--8$

$-8--6$

$-6--4$

$-4--2$

$-2-0$

$0-2$

$2-4$

4-6

$6-8$

$8-10$

- $>10$

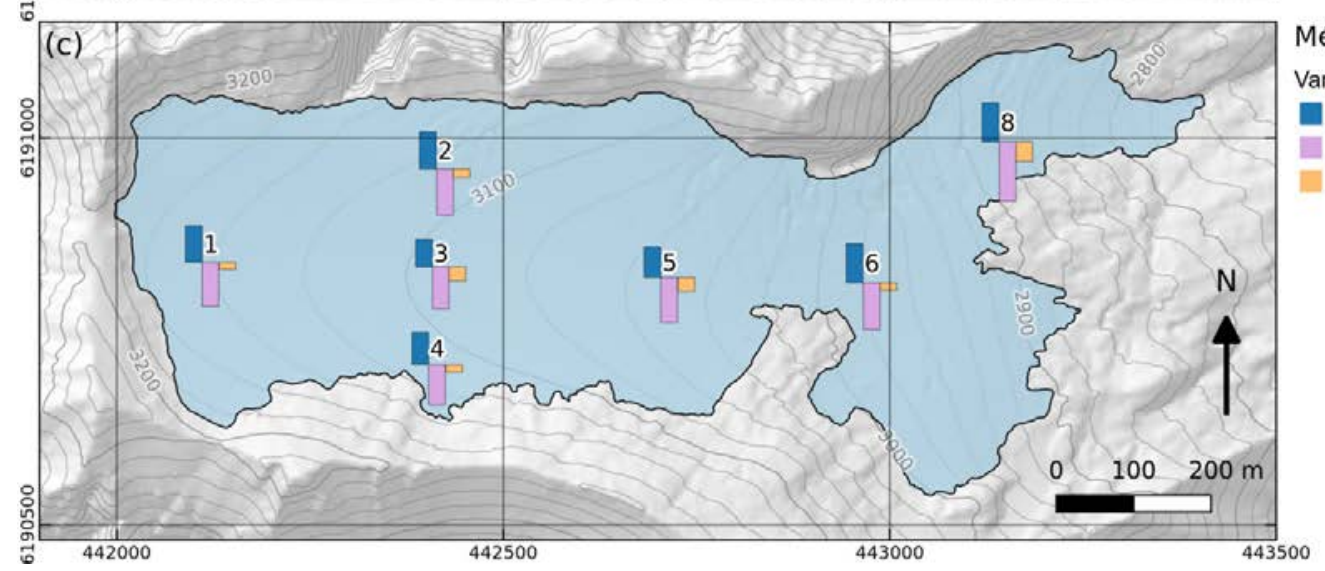

\section{Méthode glaciologique}

Variation de hauteur $(\mathrm{m})$ 2013-2017

Bilan hivernal

Bilan estival

Bilan net

Figure 4: Evolution récente du glacier d'Ossoue : (a) évolution de sa surface entre 2012 et 2017, (b) évolution de son élévation entre 2013 et 2017 par méthode géodésique (Pléiades), (c) évolution de son élévation entre 2013 et 2017 par méthode glaciologique décomposée en bilan hivernal (accumulation) et bilan estival (ablation), d'après Tableau 1.

Figure 4: Recent evolution of the Ossoue Glacier: (a) Area changes between 2012 and 2017, (b) Elevation changes between 2013 and 2017 from the geodesic method (Pléiades), (c) Elevation changes between 2013 and 2017 from the glaciological method, separated in winter mass balance (accumulation) et summer mass balance (ablation), as shown in Table 1.

+/- $1.0 \mathrm{~m}$, ce qui donne un bilan de masse de $-4,02+/-0,9$ $\mathrm{m}$ we. La carte de changement de hauteur est particulièrement bruitée pour ce glacier ce qui peut s'expliquer par le faible éclairement et la vitesse d'écoulement de ce glacier qui est particulièrement élevée. Elle a été estimée à plus de $50 \mathrm{~m} \cdot \mathrm{a}^{-1}$ dans la partie centrale du glacier à partir de caméras automatiques (Association Moraine, 2010). Pour le glacier du Petit Vignemale, la perte de hauteur est $-4,65$ $\mathrm{m}+/-1,0 \mathrm{~m}$, ce qui donne un bilan de masse de $-4,18+/-$ $0,9 \mathrm{~m}$ we (Figure 6). Toutefois il est à noter que les images Pléiades ne fournissent pas de données pour la partie haute du Petit Vignemale en raison du faible éclairage sur cette paroi. La moyenne présentée ici reflète donc seulement la moitié inférieure du glacier. 


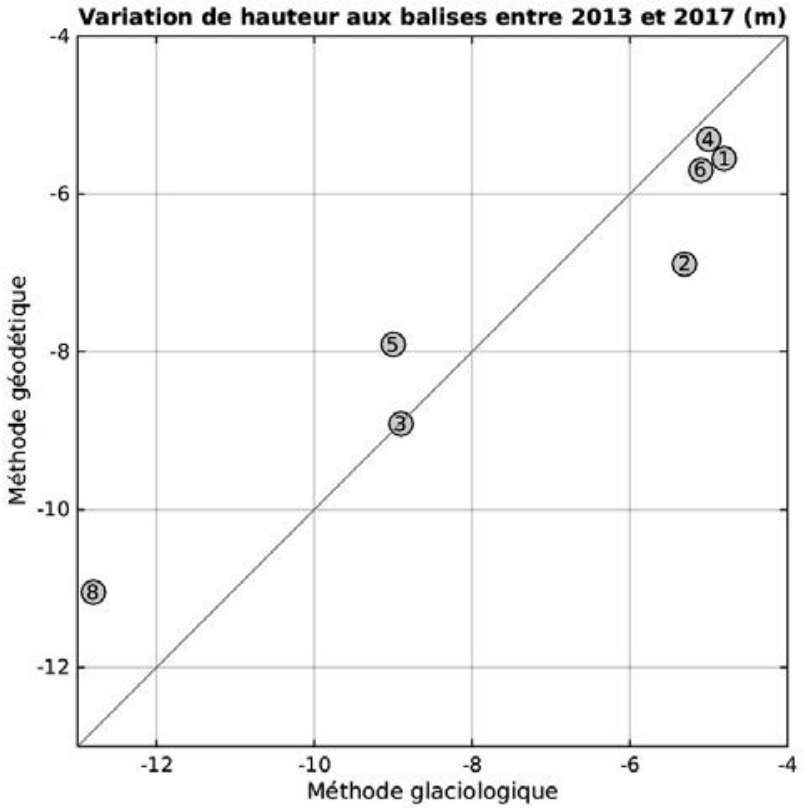

Figure 5: Comparaison des changements de hauteurs par méthode glaciologique et géodésique.

Figure 5: Comparison of elevation changes obtained from the glaciological and geodesic methods at each measurement station.

\section{Discussion}

La superficie du glacier d'Ossoue était de 44,9 ha dans l'inventaire de 2011 (Marti et al., 2015a) et de 37.2 +/- 4,9 ha dans l'inventaire de 2016 (Rico et al., 2017). La diminution de la surface du glacier d'Ossoue entre 2011 et 2016 était donc de l'ordre de 1,5 ha par an, alors qu'elle est de 4,3 ha pour la seule année hydrologique 2016-2017 à cause du détachement de la langue orientale. L'année 2016-2017 marque donc une accélération de la réduction de surface du glacier d'Ossoue, ainsi qu'un changement important de sa morphologie. Cette fragmentation est le signe d'un amincissement critique dans la partie basse du glacier.

La comparaison entre les méthodes géodésique et glaciologique montre un bon accord. En théorie, ces deux mesures ne sont pas strictement comparables puisque les observations aux balises décrivent le bilan de surface sans tenir compte de la vitesse d'émergence causée par le mouvement du glacier, alors que la méthode géodésique donne la somme des deux effets. En zone d'ablation, le bilan glaciologique doit être plus négatif que le bilan géodésique (vitesse d'émergence positive), et inversement en zone d'accumulation. Pour le glacier d'Ossoue, la dynamique glaciaire est faible comme le montrent les vitesses mesurées aux balises

\section{Elevation changes between 23 Sep 2013 and 08 Oct 2017}

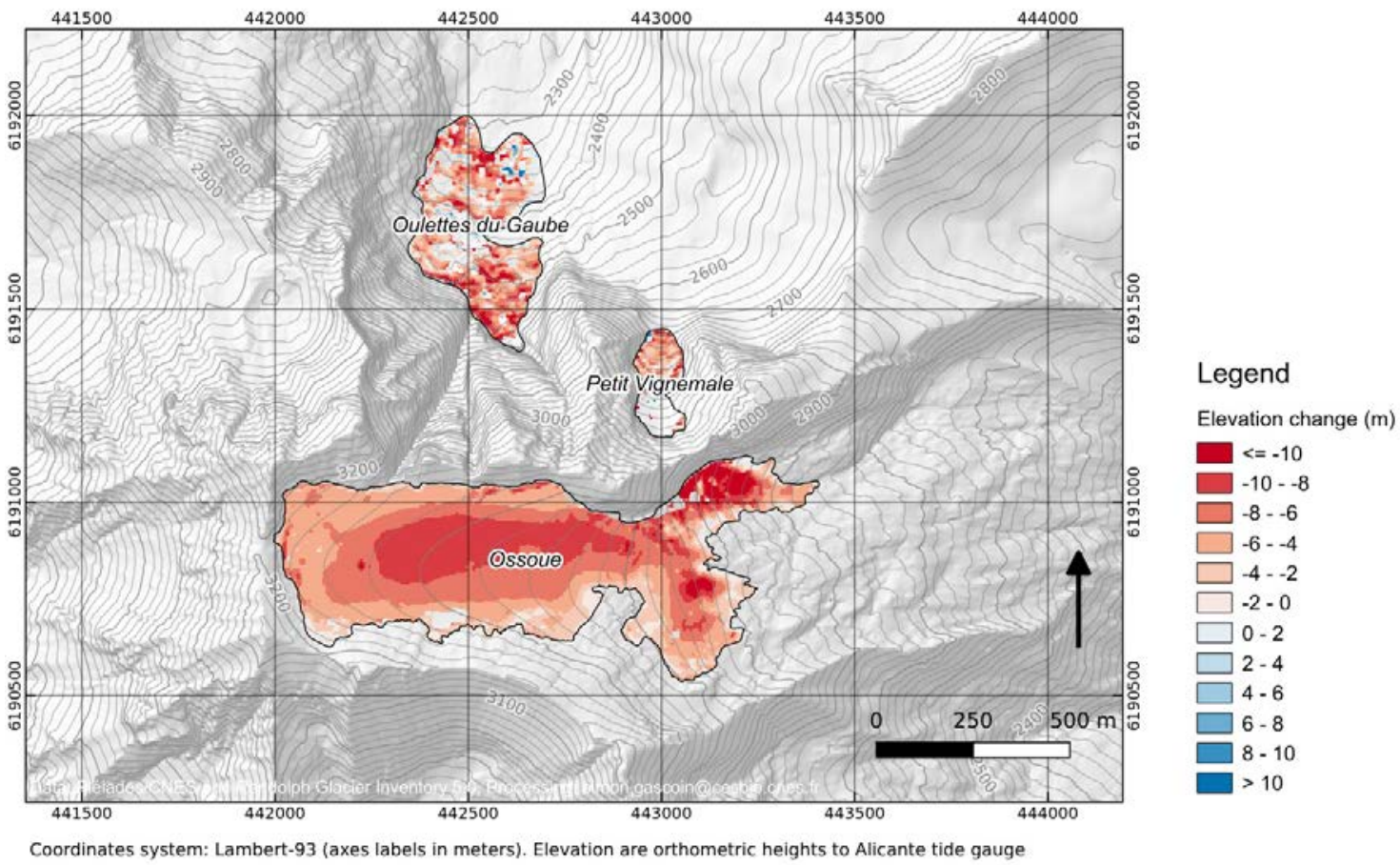

Figure 6: Carte de variation de la hauteur des glaciers du massif du Vignemale entre le 23 septembre 2013 et le 8 octobre 2017 (contour des glaciers: RGI 5.0, fond topographique : MDT05, IGN Espagne et RGE, IGN France). Les zones grises dans le haut du Petit Vignemale et la partie centrale des Oulettes sont des zones sans données.

Figure 6: Map of the elevation changes over the glaciers in the Vignemale massif between September 23, 2013 and October 8, 2017 (outline of the glaciers is from the RGI 5.0, topographic contour lines are from MDT05, IGN Spain and RGE, IGN France).

The gray areas in the upper part of the Petit Vignemale and the central part of the Oulettes are areas without data. 
Tableau 4: Vitesses de déplacement des balises sur le glacier d'Ossoue en mètres par an.

Table 4: Displacement velocities of the measurement stations on Ossoue Glacier in meters per year

\begin{tabular}{|c|c|c|c|c|c|c|}
\hline Balise & $\mathbf{2 0 0 2 - 2 0 0 3}$ & $\mathbf{2 0 0 3 - 2 0 0 5}$ & $\mathbf{2 0 0 3 - 2 0 0 6}$ & $\mathbf{2 0 0 5 - 2 0 0 6}$ & $\mathbf{2 0 1 3 - 2 0 1 5}$ & Moyenne \\
\hline 1 & 0,54 & & 0,42 & & 0,21 & 0,4 \\
\hline 2 & 0,88 & & 0,83 & & 0,32 & 0,7 \\
\hline 3 & 1,36 & 0,98 & & 1,1 & 0,46 & 1,0 \\
\hline 4 & 0,46 & 0,12 & & 0,5 & 0,12 & 0,3 \\
\hline 5 & 4,15 & & & 3,25 & 1,60 & 3,0 \\
\hline 6 & 5,39 & 3,92 & & & 1,14 & 3,5 \\
\hline 8 & 12,07 & & & & 3,49 & 7,8 \\
\hline
\end{tabular}

(Tableau 4). L'ablation est donc largement dominée par la perte d'épaisseur. Mais l'effet de l'écoulement du glacier est cohérent avec la plus faible variation de hauteur observée par la méthode géodésique au niveau de la balise 8 en particulier, où la vitesse est la plus élevée (Tableau 4; Figure 5).

A l'échelle du glacier, l'écart entre le bilan de masse par méthode géodésique $(-5,2+/-0,5 \mathrm{~m}$ we) et glaciologique $(-6,35 \mathrm{~m}$ we) peut s'expliquer par le fait que la méthode géodésique échantillonne des zones qui ont été déglacées après 2012 (année de définition de la surface du glacier), et donc contribuent à la moyenne à l'échelle du glacier par un changement d'élévation nul. Les erreurs de mesures par la méthode glaciologique, et le faible nombre de points de mesures au regard de la méthode géodésique, peuvent expliquer aussi une partie de cet écart.

Pour mettre en perspective l'évolution du glacier nous avons utilisé les données de la station météorologiques de Tarbes car cette station est la plus proche du Vignemale ayant une période de mesure suffisamment longue (également utilisée par Marti et al., 2015a). D'après cette station, la période 2013-2017 serait plutôt chaude et arrosée dans cette région des Pyrénées. Les températures minimales et maximales enregistrées à la station de Tarbes furent généralement plus élevées que la médiane de la période 1960-2016 (Figure 7). En particulier, la fin de l'année 2014 fut exceptionnellement chaude (maximum de la période de référence). Les précipitations hivernales furent généralement supérieures à la médiane en 2014, 2015 et 2016 à Tarbes. Si on fait l'hypothèse que ces précipitations sont représentatives de l'accumulation sur le massif du Vignemale, cela montre qu'une accumulation hivernale abondante est insuffisante pour compenser la fonte au cours de la saison d'ablation. Toutefois, il est probable, comme l'expliquent López-Moreno et al. (2016) dans le cas du glacier Monte Perdido, que les variations climatiques récentes aient un impact limité sur l'évolution du glacier car celui-ci est largement en déséquilibre avec le climat local depuis la fin des années 1990. De plus, la réduction de la taille du glacier peut entrainer une rétroaction positive à travers l'émission de rayonnement thermique depuis les parois récemment déglacées (López-Moreno et al., 2016).
La structure spatiale de la variation de hauteur du glacier d'Ossoue est similaire à celle déjà observée sur la période 1983-2013 (Marti et al., 2015a): le centre du Plateau des Neiges s'est aminci plus rapidement que sa périphérie (Figure 4). Les mesures de balises suggèrent que cela est dû à un contraste d'accumulation (Figure 4). Il est possible que la périphérie du plateau accumule davantage de neige au cours de l'hiver en raison de phénomènes locaux :

(i) transport de la neige par le vent d'ouest depuis le versant espagnol vers le secteur situé à proximité du col de Cerbillona (balise 1). Le même phénomène pourrait expliquer l'accumulation plus élevée en aval de la rupture de pente du glacier (balise 6) ;

(ii) transport gravitationnel de la neige depuis les parois du Vignemale et du Montferrat qui sont trop pentues pour accumuler le manteau neigeux (balises 2, balise 4).

(iii) En outre, l'effet d'ombrage le long de la crête du Montferrat peut aussi contribuer à limiter d'éventuelles périodes de fonte qui ont lieu avant la mesure d'accumulation. Avec le creusement du glacier, la rive droite du glacier se retrouve davantage exposée au nord et donc reçoit moins d'énergie solaire ; ce qui explique le positionnement de la zone de perte de masse maximale par rapport au talweg du glacier.

Les bilans de masse par méthode géodésique et glaciologique sont cohérents et indiquent que glacier d'Ossoue a perdu de la masse à une cadence proche de $1,5 \mathrm{~m}$ we. $\mathrm{a}^{-1}$. Bien que ce recul s'inscrive dans la tendance générale de retrait des glacier pyrénéens (López-Moreno et al., 2016; Rico et al., 2107), cette valeur est significativement plus négative que celle mesurée sur le glacier Monte Perdido entre 2011 to $2014\left(-0.58 \pm 0.36 \mathrm{~m}\right.$ we. $\mathrm{a}^{-1}$, López-Moreno et al., 2016). A titre de comparaison, son bilan de masse est hors de l'intervalle formé par un écarttype autour de la moyenne des bilans de masse des 40 glaciers de référence du WGMS durant la période 2015 2016 (WGMS, 2017). En supposant une distribution normale des bilans de masse annuels, et en supposant que la 

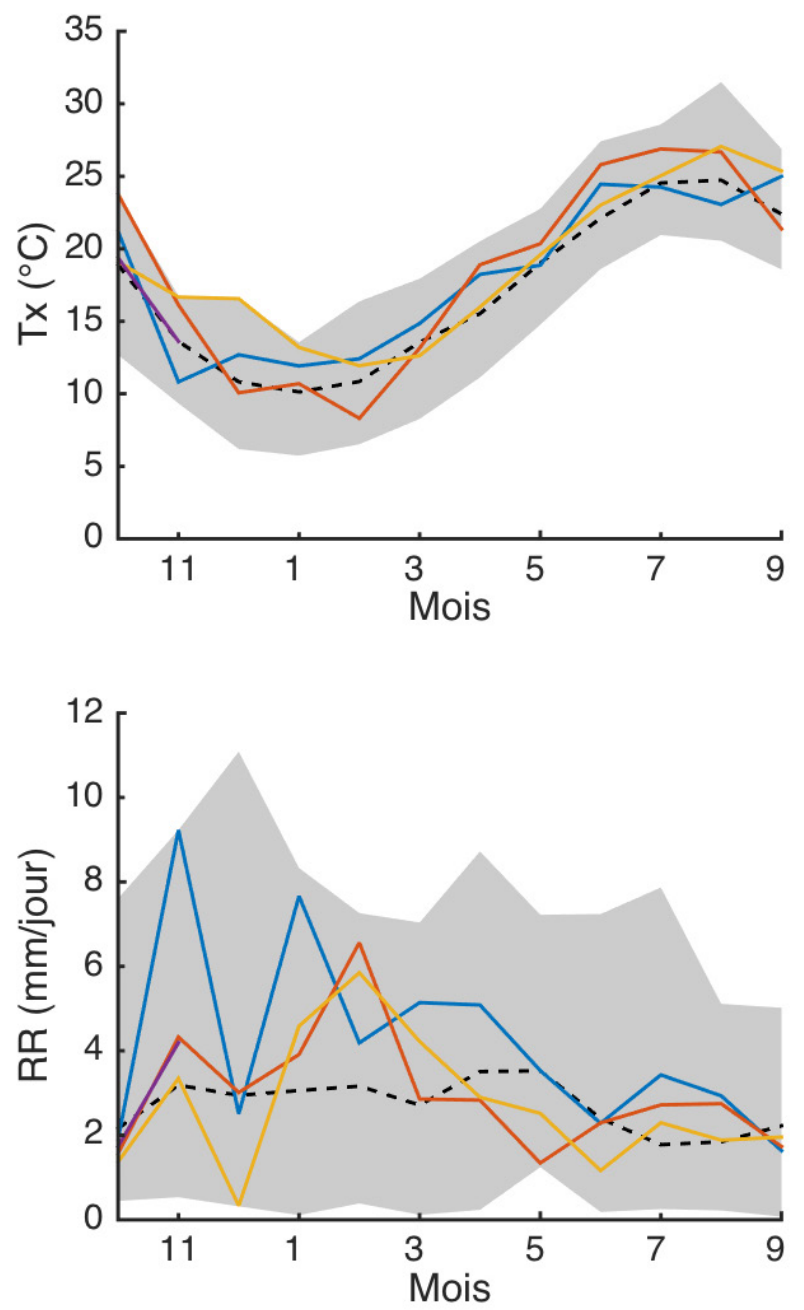
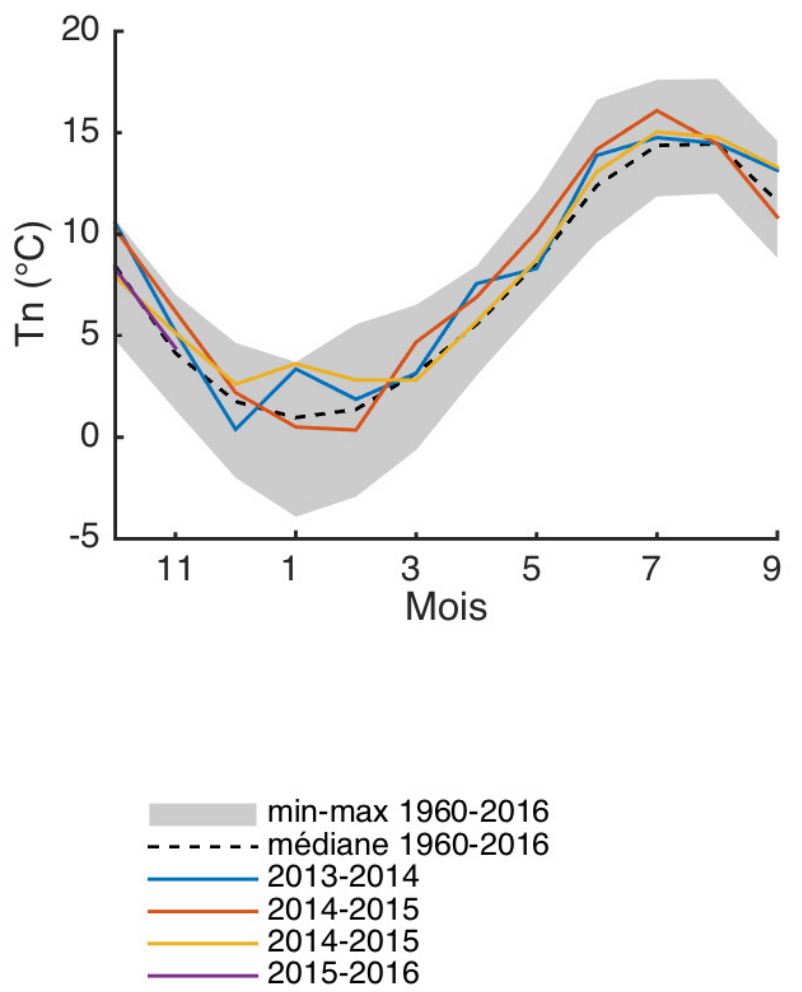

Figure 7: Evolution des conditions météorologiques à la station de Tarbes (source: Météo-France) entre octobre 2013 et novembre

2016 et comparaison avec la climatologie sur la période 1960-2016. Les graphiques présentent les moyennes mensuelles des variables: température de l'air maximale journalière (Tx), température de l'air minimale journalière (Tn).et précipitations ( $\mathrm{mm} / \mathrm{jour})$. Figure 7: Evolution of weather conditions at Tarbes station (source: Mété-France) between October 2013 and November 2016 and

comparison with climatology over the period 1960-2016. The graphs present the monthly averages of the maximum daily air temperature (Tx), minimum daily air temperature (Tn), and precipitation ( $\mathrm{mm} /$ day).

population de référence du WGMS représente bien l'ensemble des glaciers du globe, cela signifie que le glacier d'Ossoue fait partie des $15 \%$ des glaciers dont le rythme de perte de masse est le plus élevé sur Terre.

\section{Conclusion}

Nous avons présenté une mise à jour du bilan de masse du glacier d'Ossoue à partir d'images Pléiades pour la période 2013-2017. Cette étude permet de prolonger celle de Marti et al. (2015a) et nous confirme que l'amincissement du glacier d'Ossoue n'a pas ralenti au cours des quatre dernières années. D'après les archives disponibles, ce recul qui a démarré dans les années 1980 est sans précédent depuis la fin du Petit Âge glaciaire. Les données Pléiades révèlent que les deux autres glaciers du massif du Vignemale ont une perte de masse spécifique similaire sur la période 2013-2017. En revanche la superficie du Petit Vignemale est stable.

Le glacier d'Ossoue s'amincit plus rapidement au centre du Plateau des Neiges conformément aux mesures glaciologiques effectuées par l'association Moraine. Une étude plus poussée est nécessaire pour déterminer les facteurs de ce patron d'ablation, en particulier une perspective de ce travail serait d'évaluer l'importance du transport de la neige par le vent et par les avalanches dans la redistribution spatiale du manteau neigeux.

Cette étude montre que les progrès en télédétection optique à haute résolution permettent d'envisager un suivi régulier de petits glaciers de montagne comme le glacier d'Ossoue (32 ha). Dans le cas du glacier d'Ossoue, où les changements d'élévation de la surface sont supérieurs à $1 \mathrm{~m} \cdot \mathrm{a}^{-1}$, un DEM dérivé d'images stéréoscopiques 
Pléiades serait suffisamment précis pour détecter les changements d'élévation d'une année à l'autre. Les mesures de terrain restent toutefois indispensables, en particulier pour interpréter les changements observés en termes de bilan de masse saisonnier.

\section{Remerciements}

Ce travail s'inscrit dans le cadre du projet européen Interreg-POCTEFA CLIM'PY de l'Observatoire Pyrénéen du Changement Climatique (OPCC). Ce travail a été soutenu par le Programme National de Télédétection Spatiale (PNTS, http://www.insu.cnrs.fr/pnts), projet $n^{\circ}$ PNTS-2018-4. Ce travail a bénéficié du soutien du CNES à travers le projet TOSCA “Observatoire Spatial de l'Enneigement" et le programme ISIS d'acquisition d'images Pléiades. L'association Moraine est soutenue par le conseil départemental de la Haute-Garonne, le conseil départemental des Hautes-Pyrénées, le conseil régional Occitanie et le Parc National des Pyrénées. Les auteurs remercient également Etienne Berthier et Mauri Pelto pour leurs conseils durant la rédaction de cet article. Cette étude n'aurait pas vu le jour sans le travail de doctorat de Renaud Marti (financement Région Midi-Pyrénées et Université de Toulouse), que les auteurs remercient ici.

\section{Références}

Association Moraine, 2010. Le glacier des Oulètes de Gaube (Vignemale), Surveillance à partir d'appareils photos automatiques (2009), rapport d'étude, 2010, 9 pp.

Arendt, A., Bliss, A., Bolch, T., Cogley, J.G., Gardner, A.S., Hagen, J.O., ... \& Pfeffer, W.T., 2015. Randolph glacier inventory-a dataset of global glacier outlines: version 5.0. Global Land Ice Measurements from Space; Digital Media: Boulder, CO, USA.

Belart, J.M., Berthier, E., Magnússon, E., Anderson, L.S., Pálsson, F., Thorsteinsson, T., Howat, I.M., Aðalgeirsdóttir, G., Jóhannesson, T. and Jarosch, A.H., 2017. Winter mass balance of Drangajökull ice cap (NW Iceland) derived from satellite sub-meter stereo images. The Cryosphere, 11(3): 1501-1517. doi: 10.5194/tc-11-1501-2017

Berthier, E., Arnaud, Y., Kumar, R., Ahmad, S., Wagnon, P. \& Chevallier, P., 2007. Remote sensing estimates of glacier mass balances in the Himachal Pradesh (Western Himalaya, India). Remote Sensing of Environment, 108(3): 327-338. https://doi.org/10.1016/j.rse.2006.11.017
Berthier, E., Vincent, C., Magnússon, E., Gunnlaugsson, Á.P., Pitte, P., Le Meur, E., ... \& Wagnon, P., 2014. Glacier topography and elevation changes derived from Pléiades sub-meter stereo images. The Cryosphere, 8(6): 2275-2291. https://doi.org/10.5194/tc-8-2275-2014

Gleyzes, M.A., Perret, L. \& Kubik, P., 2012. Pleiades system architecture and main performances. International Archives of the Photogrammetry, Remote Sensing and Spatial Information Sciences, 39(1): 537-542. https://doi.org/10.5194/ isprsarchives-XXXIX-B1-537-2012

Höhle, J. \& Höhle, M., 2009. Accuracy assessment of digital elevation models by means of robust statistical methods. ISPRS Journal of Photogrammetry and Remote Sensing, 64(4): 398406. https://doi.org/10.1016/j.isprsjprs.2009.02.003

López-Moreno, J.I., Revuelto, J., Rico, I., Chueca-Cía, J., Julián, A., Serreta, A., Serrano, E., Vicente-Serrano, S.M., Azorín-Molina, C., Alonso-González, E. \& García-Ruiz, J.M., 2016. Thinning of the Monte Perdido Glacier in the Spanish Pyrenees since 1981. The Cryosphere, 10: 681-694. https://doi.org/10.5194/tc-10-681-2016

Marti, R., Gascoin, S., Houet, T., Laffly, D. \& René, P., 2014. Evaluation du modèle numérique d'élévation d'un petit glacier de montagne généré à partir d'images stéréoscopiques Pléiades: cas du glacier d'Ossoue, Pyrénées françaises. Revue Française de Photogrammétrie et de Télédétection, (208): 57-62.

Marti, R., Gascoin, S., Houet, T., Ribière, O., Laffly, D., Condom, T., ... \& Soubeyroux, J.M., 2015a. Evolution of Ossoue Glacier (French Pyrenees) since the end of the Little Ice Age. The Cryosphere, 9(5): 1773-1795. https://doi. org/10.5194/tc-9-1773-2015

Marti, R., Gascoin, S., Houet, T., Laffly, D. \& René, P., 2015 b. The Pyrenean glaciers (South West Europe) in 1850 and 2011: a new cross-border inventory based on aerial images and field surveys. In 26th IUGG General Assembly.

Marti, R., Gascoin, S., Berthier, E., De Pinel, M., Houet, T. \& Laffly, D., 2016. Mapping snow depth in open alpine terrain from stereo satellite imagery. The Cryosphere, 10(4): 1361. https://doi.org/10.5194/tc-10-1361-2016

René, P., 2011. Régression des glaciers pyrénéens et transformation du paysage depuis le Petit Âge glaciaire. Sud-Ouest Européen. Revue Géographique des Pyrénées et du Sud-Ouest, (32): 5-19. https://doi.org/10.4000/soe.639

René, P., 2013. Glaciers des Pyrénées: le réchauffement climatique en images. Éd. Cairn.

René, P., 2014. Le suivi des glaciers dans les Pyrénées françaises. Météorologie, (85): 27-34. https://doi.org/10.4267/2042/53748

Rico, I., Izagirre, E., Serrano, E. \& López-Moreno, J.I., 2017. Superficie glaciar actual en los Pirineos: Una actualización para 2016. Pirineos, 172, e029. doi: https://dx.doi. org/10.3989/Pirineos.2017.172004

WGMS, 2017. Fluctuations of Glaciers Database. World Glacier Monitoring Service, Zurich, Switzerland. doi: 10.5904/ wgms-fog-2017-10. 